\title{
Analysis of Output Capacitor Voltage Ripple in Multi-Phase Transformer-Linked Boost Chopper Circuit
}

\author{
Jun Imaoka* \\ Yuta Nakamura** Member, \\ $\begin{array}{ll}\text { Masayoshi Yamamoto* }^{*} & \text { Member } \\ \text { Takahiro Kawashima** }^{* *} & \text { Member }\end{array}$
}

(Manuscript received Dec. 28, 2012, revised April 6, 2013)

\begin{abstract}
The multi-phase method is effective in downsizing the power converter system. Because of the multi-phase boost chopper circuit, the output capacitor and the cooling system can be downsized in the case of the boost chopper circuit. Furthermore, the volume of the inductor can be reduced using the transformer-linked method. However, there is little comparative data available on the output capacitor in the boost chopper of the conventional multi-phase method and the transformer-linked method. The purpose of my work was to examine the characteristics of the output capacitor in both the multi-phase method and the transformer-linked method. To evaluate the performance of the downsized capacitor, a voltage ripple analysis has been successfully employed as the index of the volume of the output capacitor. From the experimental results, it was found that there is little difference between the multi-phase method and the transformer-linked method in regard to the voltage ripple of the output capacitor. The experimental results indicate that the performance of the downsized capacitor is the same for the multi-phase method and the transformer-linked method.
\end{abstract}

Keywords: multi-phase, boost chopper circuit, miniaturization of output capacitor

\section{Introduction}

Recent interest in the miniaturization of the SMPS, the power supply in the consumer electronics and the power converter in Hybrid Electric Vehicle (HEV) and Electric Vehicle $(\mathrm{EV})$ has spurred a great deal of research into the multiphase techniques for the buck and boost chopper system. It is well known that the multi-phase method has a couple of effectiveness for the miniaturization technique. One is the miniaturized cooling system. The other is the miniaturized output capacitor in the buck and boost chopper. In addition to this, the transformer-linked method can achieve the miniaturization of the inductor volume in the multi-phase power converter system $^{(1)-(5)}$. And the several design method for the coupled inductor of the transformer-linked method has been proposed to achieve the high power density performance in the power converter system ${ }^{(6)-(10)}$. When the coupled inductor is designed on the basis of the method that is shown in reference ${ }^{(12)}$, the coupled inductor can be miniaturized to approximately half size as compared with the non-coupled inductors. In fact, Fig. 1 shows the comparison result of the inductor size of the transformer-linked and non-coupled methods in multi-phase boost converter. The experiment was evaluated under the condition of the same inductor ripple current and flux density between the transformer-linked and the noncoupled inductors. From this result, the effectiveness of the

\footnotetext{
Shimane University

1060, Nishikawatsu, Matsue, Shimane 690-8504, Japan

** DENSO CORPORATION

1-1, Showa-cho, Kariya, Aichi 448-8661, Japan

*** Shimane Institute for Industrial Technology

1, Hokuryo-cho, Matsue, Shimane 690-0816, Japan
}



Fig. 1. Comparison result of the inductor size of the transformer-linked and non-coupled methods in the multi-phase boost converter

transformer-linked method boost converter is clear in terms of compact, lightweight and the lower cost points of view.

However, there is little comparative discussion of the capacitor size in both the conventional multi-phase method (non-coupled method) and the transformer-linked method.

In this study, the capacitor size of the multi-phase noncoupled method and the transformer-linked method has been discussed by using the voltage ripple analysis of the output capacitor in the boost chopper system. Furthermore, the comparative data of the capacitor size between the noncoupled method and the transformer-linked method are evaluated from the experimental point of view.

\section{Characteristic Analysis Method}

Main factors which determine a capacitor size are capacitance value and withstand voltage. Withstand voltage is decided by an output voltage's specification of the circuit. On the other hand, all capacitance value can be applied to a circuit if the output ripple voltage is suppressed within the 
standard of it. Therefore, the possibility of downsizing is discussed with how much the capacitance value decreases in this paper. An equation (1) shows the relational equation among capacitance value $C$, voltage $V_{\mathrm{c}}$ which is generated on the capacitor, and charge $Q_{\mathrm{c}}$.

$$
V_{\mathrm{c}}=\frac{Q_{\mathrm{c}}}{C}
$$

It is understood from above equation that ripple voltage which is generated on a capacitor is determined by capacitance value $C$ and charge $Q_{\mathrm{c}}$. Ripple voltage $\Delta V_{\mathrm{c}}$ is a parameter which decides a circuit performance so that it is suppressed until the standard value generally. Hence, the range of ripple voltage $\Delta V_{\mathrm{c}}$ is possible to evaluate the capacitance value $C$ by the range of the charge fluctuation $\Delta Q_{\mathrm{c}}$. Therefore, first of all, it is required to analysis charge fluctuation $\Delta Q_{\mathrm{c}}$ of an output capacitor for discussing downsizing of a capacitor. Charge is calculated from time waveform's density of capacitor current because of time integration of current.

However, current waveforms of an output capacitor depend on a condition such as inductor current or duty ratio $d$. It means that a part of calculating density for charge differs. For that reason, by adapting the change of waveform, it is needed to change the equation to calculate the density.

\section{Characteristic Analysis of Single-phase Method}

A charge characteristic analysis of the output smoothing capacitor of the single-phase method, which is shown in Fig. 2, is carried out in this section. The charge quantity of when the capacitor current is positive is equal to the one of when the capacitor current is negative by discharge and charge equilibrium condition of a capacitor. For that reason, the quantity of the charge fluctuation can be calculated by means of the calculating the charge quantity of the discharge or charge time in case of analyzing charge fluctuation. Assuming that the diode current is $i_{\mathrm{d}}$, the current waveform of the output smoothing capacitor should be described as Fig. 3. The current $I_{\mathrm{dp}}$ and $I_{\mathrm{Lpp}}$ show the peak value of the diode current and ripple value of the inductor current respectively. In this time, each peak value of the output smoothing capacitor current is expressed as

$$
\begin{aligned}
& I_{\mathrm{c} \_\mathrm{A} \_\sin }=I_{\mathrm{dp}}-I_{\mathrm{o}} \ldots \ldots \ldots \ldots \ldots \ldots \ldots \ldots \ldots \ldots \ldots \ldots \ldots \ldots \ldots \ldots \ldots \ldots
\end{aligned}
$$

And also, the charge quantity of the discharge and charge time can be calculated from these equations of the peak value. At this point, the time integrated values of the current $i_{\mathrm{d}}$ which is supplied from the diode and current which is outputted to the load for one switching cycle became equal. That's why the following equation is derived.

$$
\frac{1}{2} \cdot\left(I_{\mathrm{dp}}+I_{\mathrm{dp}}-I_{\mathrm{Lpp}}\right) \cdot T_{\mathrm{off}}=I_{\mathrm{o}} \cdot T_{\mathrm{s}}
$$

On the other hand, because the peak value of the diode current $I_{\mathrm{dp}}$ and relational equation of the average inductor current $I_{\mathrm{L}}$, and $I_{\mathrm{Lpp}}$ is expressed as the equation (5), the output current $I_{\mathrm{O}}$ is described as equation (6) from (4) and (5).

$$
I_{\mathrm{dp}}=I_{\mathrm{L}}+\frac{1}{2} \cdot I_{\mathrm{Lpp}}
$$



Fig. 2. Single-phase boost chopper circuit



Fig. 3. Current waveforms of the single-phase boost chopper circuit



Form 1



Form 2
Fig. 4. Output capacitor current waveforms of the single-phase boost chopper circuit

$$
I_{\mathrm{o}}=I_{\mathrm{L}} \cdot(1-d)
$$

In case of single-phase method, there are two patterns of the current waveforms which flow through the output smoothing capacitor as seen in Fig. 4. According to the waveforms patterns, the quantity of the charge fluctuation on the discharge and charge time is calculated by selecting the field for being easy. From above reason, the case which shows a triangle shape waveform is defined as Form1. And also, the case which shows a rectangle shape's waveform is defined as Form 2 as well. From $I_{\mathrm{c}_{-} \mathrm{B}_{-} \sin }<0$, a range of the duty ratio in case of Form 1 is given by

$$
d_{\text {sin_ } 1}<\frac{1}{2} \cdot \frac{I_{\text {Lpp_sin }}}{I_{\mathrm{L}_{-} \sin }}
$$

The condition which the waveform changes is determined by the ratio of the average inductor current $I_{\mathrm{L}_{-} \sin }$ to the ripple current's amplitude $I_{\mathrm{Lpp}_{-} \sin }, I_{\mathrm{Lpp}_{-} \sin } / I_{\mathrm{L}_{-} \sin }$. From $I_{\mathrm{c}_{-} \mathrm{B} \_ \text {sin }} \geq 0$, a range of the duty ratio in case of Form 2 is

$$
d_{\text {sin_2 }} \geq \frac{1}{2} \cdot \frac{I_{\text {Lpp_sin }}}{I_{\mathrm{L}_{-} \sin }}
$$

The range of the charge fluctuation $\Delta Q_{\mathrm{c}_{-} \sin _{-} 1}$ is described as following by calculating the square of a triangle part at a range of the equation (7) on Form1 in Fig. 4.

$$
\Delta Q_{\mathrm{C}_{-} \sin \_1}=\frac{1}{2} \cdot\left(d+\frac{1}{2} \cdot \frac{I_{\mathrm{Lpp} \__{-} \sin }}{I_{\mathrm{L}_{-} \sin }}\right)^{2} \cdot(1-d) \cdot \frac{I_{\mathrm{L}_{-} \sin }^{2}}{I_{\mathrm{Lpp}_{-} \sin }} \cdot T_{\mathrm{s}}
$$


Table 1. Circuit parameters (single-phase)

\begin{tabular}{lc|c}
\hline \hline Input voltage & $V_{\mathrm{i}}$ & $50 \mathrm{~V}$ \\
\hline Duty ratio & $d$ & $0.1 \sim 0.7$ \\
\hline Output capacitance & $C_{\mathrm{o}}$ & $3.9 \mu \mathrm{F}$ \\
\hline Self inductance & $L$ & $300 \mu \mathrm{H}$ \\
\hline Output resistance & $R_{\mathrm{o}}$ & $48 \Omega$ \\
\hline Switching frequency & $f_{\mathrm{s}}$ & $50 \mathrm{kHz}$ \\
\hline
\end{tabular}

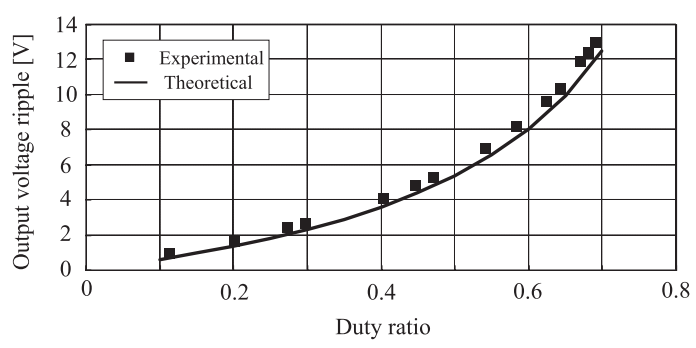

Fig. 5. Output voltage ripple of the single-phase boost chopper circuit

Likewise, the range of the charge fluctuation $\Delta Q_{\mathrm{c}_{-} \sin _{-} 2}$ is represented as following by calculating the square of a rectangle part at a range of the equation (8) on Form2 in Fig. 4.

$$
\Delta Q_{\mathrm{C}_{-} \sin \_2}=\frac{I_{\mathrm{Lpp}_{\_} \sin }}{I_{\mathrm{L}_{-} \sin }} \cdot d \cdot(1-d) \cdot \frac{I_{\mathrm{L}_{-} \sin }^{2}}{I_{\mathrm{Lpp}_{-} \sin }} \cdot T_{\mathrm{s}}
$$

Now, the output ripple voltage is measured and the validity of the derived equation is discussed from the experimental point of view. The measurement value from the experiment is compared with the theoretical value of the ripple voltage with the following equation (11).

$$
\Delta V_{\mathrm{c}}=\frac{\Delta Q_{\mathrm{c}}}{C_{\mathrm{o}}}
$$

The circuit parameters are shown in Table 1. The theoretical value of the output ripple voltage for the duty ratio from 0.1 to 0.7 is depicted as a solid line in Fig. 5. The measurement result of the output voltage ripple from the experiment with the same circuit parameter is plotted in Fig. 5. As a result, the theoretical value agrees with the measurement value in close proximity, and hence the validity of the calculation equation regarding the quantity of the charge fluctuation $\Delta Q_{\mathrm{c}}$ is verified.

\section{Characteristic Analysis of Multi-phase Method}

In this section, a charge characteristic analysis of the output smoothing capacitor of a non-coupled multi-phase boost chopper circuit in Fig. 6 is carried out. In case of the multiphase method, the characteristic of the output capacitor current $i_{\mathrm{c}}$ changes on the border of the duty ratio 0.5 because each phase of the current overlaps each other in the output side. Accordingly, separating the cases of $d<0.5$ and $d \geq$ 0.5 is required on analysis. Furthermore, each phase of the variation related to parasitic parameters doesn't exist when analyzing and also, each phase of the average current is assumed to be equal.

First, the case of $d<0.5$ is investigated. Each phase of



Fig. 6. Non-coupled multi-phase boost chopper circuit



Fig. 7. Current waveforms of the non-coupled multiphase boost chopper circuit $(d<0.5)$

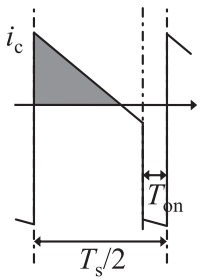

Form 1



Form 2

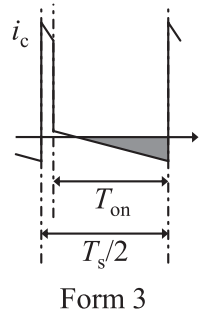

Fig. 8. Output capacitor current waveforms of the noncoupled multi-phase boost chopper circuit $(d<0.5)$

the diode current is defined as $i_{\mathrm{d} 1}$ and $i_{\mathrm{d} 2}$ respectively, and then the current waveforms of the output capacitor in case of $d<0.5$ is shown in Fig. 7. As depicted in Fig. 7, it is understood that the output capacitor current which each phase of the diode current overlaps is generated under the condition of $d<0.5$. In this time, each peak value of the output capacitor current is given by

$$
\begin{aligned}
& I_{\mathrm{c} \_\mathrm{A} \_ \text {mul }}=2 I_{\mathrm{dp}}-\frac{I_{\mathrm{Lpp}}}{T_{\mathrm{off}}} \cdot \frac{T_{\mathrm{s}}}{2}-I_{\mathrm{O}} \\
& I_{\mathrm{c} \_ \text {B } \_ \text {mul }}=2 I_{\mathrm{dp}}-I_{\mathrm{Lpp}}-\frac{I_{\mathrm{Lpp}}}{T_{\text {off }}} \cdot\left(\frac{T_{\mathrm{s}}}{2}-T_{\mathrm{on}}\right)-I_{\mathrm{o}} \cdots \\
& I_{\text {c_C_mul }}=I_{\text {dp }}-\frac{I_{\mathrm{Lpp}}}{T_{\text {off }}} \cdot\left(\frac{T_{\mathrm{s}}}{2}-T_{\text {on }}\right)-I_{\mathrm{o}} \\
& I_{\text {c_D_mul }}=I_{\text {dp }}-\frac{I_{\mathrm{Lpp}}}{T_{\text {off }}} \cdot \frac{T_{\mathrm{s}}}{2}-I_{\mathrm{o}}
\end{aligned}
$$

Where, $I_{\mathrm{dp}}$ is the peak diode current, $I_{\mathrm{Lpp}}$ is the ripple current of the inductor. The charge quantity of the discharge and charge time is delivered with these equations of the peak value. The time integrated value of the output current $I_{\mathrm{o}}$ is equal to the sum of each phase of the diode current $i_{\mathrm{d} 1}$ and $i_{\mathrm{d} 2}$ 
for one switching cycle. Hence,

$$
2 \cdot \frac{1}{2} \cdot\left(I_{\mathrm{dp}}+I_{\mathrm{dp}}-I_{\mathrm{Lpp}}\right) \cdot T_{\mathrm{off}}=I_{\mathrm{o}} \cdot T_{\mathrm{s}}
$$

On the other hand, the relational expression of peak diode current value $I_{\mathrm{dp}}$, average inductor current $I_{\mathrm{L}}$, and inductor ripple current $I_{\text {Lpp }}$ are described as

$$
I_{\mathrm{dp}}=I_{\mathrm{L}}+\frac{1}{2} \cdot I_{\mathrm{Lpp}}
$$

Using the equations (16), (17), the output current $I_{\mathrm{o}}$ is expressed as

$$
I_{\mathrm{o}}=2 \cdot I_{\mathrm{L}} \cdot(1-d)
$$

From same point of view concerning single-phase method, the waveform is separated to three patterns, Form1, Form2 and Form 3 as shown Fig. 8. Form1 is given as the case that a calculation part of the positive current is a triangle shape, Form 2 is defined that the case of a calculation part of the positive current is a trapezoid shape, and Form 3 is given as the case that a calculation part of the negative current is a triangle shape respectively. In case of Form1, a range of the duty ratio, $d_{\text {mul_d }<0.5 \_1}$ is as follows from $I_{\text {c_B_mul }}<0$.

$$
d_{\text {mul_d }<0.5 \_1}<\frac{1}{2}+\frac{1}{4} \cdot \frac{I_{\text {Lpp_mul }}}{I_{\text {L_mul }}}-\frac{1}{4} \cdot \sqrt{4+\left(\frac{I_{\text {Lpp_mul }}}{I_{\text {L_mul }}}\right)^{2}}
$$

The condition which the waveform changes is determined by the ratio $I_{\text {Lpp_mul }} / I_{\mathrm{L} \_m u l}$ of the average inductor current $I_{\mathrm{L} \_m u l}$ and the ripple current's amplitude $I_{\text {Lpp_mul }}$. In case of Form3, a range of the duty ratio, $d_{\text {mul_d }<0.5 \_3}$ is as follows from $I_{\text {c_C_mul }} \geq 0$

$$
\begin{aligned}
d_{\text {mul_d }<0.5 \_3} \geq & \frac{3}{4}+\frac{1}{8} \cdot \frac{I_{\text {Lpp_mul }}}{I_{\text {L_mul }}} \\
& -\frac{1}{8} \cdot \sqrt{4+12 \cdot \frac{I_{\text {Lpp_mul }}}{I_{\text {L_mul }}}+\left(\frac{I_{\text {Lpp_mul }}}{I_{\text {L_mul }}}\right)^{2}}
\end{aligned}
$$

A range of Form 2 is in the field between Form 2 and Form3. Therefore, a range of the duty ratio, $d_{\text {mul_d }<0.5 \_2}$ is given by

$$
\begin{aligned}
& \frac{1}{2}+\frac{1}{4} \cdot \frac{I_{\text {Lpp_mul }}}{I_{\text {L_mul }}}-\frac{1}{4} \cdot \sqrt{4+\left(\frac{I_{\text {Lpp_mul }}}{I_{\text {L_mul }}}\right)^{2}} \leq d_{\text {mul_d }<0.5 \_2} \\
& <\frac{3}{4}+\frac{1}{8} \cdot \frac{I_{\text {Lpp_mul }}}{I_{\text {L_mul }}}-\frac{1}{8} \cdot \sqrt{4+12 \cdot \frac{I_{\text {Lpp_mul }}}{I_{\text {L_mul }}}+\left(\frac{I_{\text {Lpp_mul }}}{I_{\text {L_mul }}}\right)^{2}}
\end{aligned}
$$

Next, the quantity of the charge fluctuation $\Delta Q_{\mathrm{c}}$ is derived with these three ranges. In the range of equation (19) where the waveform of Form 1 is formed, the quantity of the charge fluctuation $\Delta Q_{\text {c_mul_d }<0.5 \_1}$ is expressed as

$$
\begin{aligned}
\Delta Q_{\mathrm{C} \_ \text {mul_d }<0.5 \_1}= & \frac{1}{4} \cdot\left(2 \cdot d+\frac{1}{2} \cdot \frac{1-2 \cdot d}{1-d} \cdot \frac{I_{\mathrm{Lpp} \_\mathrm{mul}}}{I_{\mathrm{L} \_\mathrm{mul}}}\right)^{2} \\
& \cdot(1-d) \cdot \frac{I_{\mathrm{L} \_\mathrm{mul}}^{2}}{I_{\mathrm{Lpp} \_\mathrm{mul}}} \cdot T_{\mathrm{s}} \cdots \cdots(22)
\end{aligned}
$$



Fig. 9. Current waveforms of the non-coupled multiphase boost chopper circuit $(d \geq 0.5)$

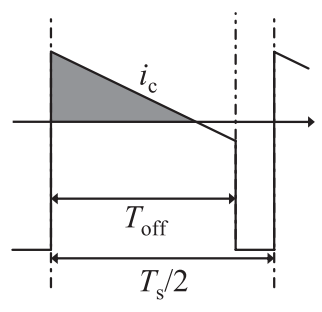

Form 1

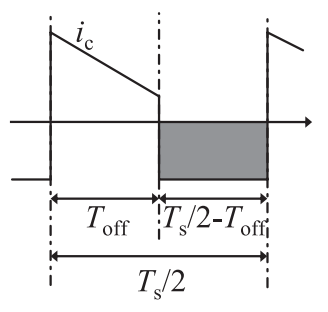

Form 2
Fig. 10. Output capacitor current waveforms of the noncoupled multi-phase boost chopper circuit $(d \geq 0.5)$

Likewise, in the range of equation (21) where the waveform of Form 2 is formed, the quantity of the charge fluctuation $\Delta Q_{\text {c_mul_d }<0.5 \_2}$ is represented as

$$
\Delta Q_{\text {C_mul_d }<0.5 \_2}=\frac{I_{\text {Lpp_mul }}}{I_{\text {L_mul }}} \cdot(1-2 \cdot d) \cdot d \cdot \frac{I_{\text {L_mul }}^{2}}{I_{\text {Lpp_mul }}} \cdot T_{\mathrm{s}}
$$

And also, in the range of equation (20) where the waveform of Form3 is formed, the quantity of the charge fluctuation $\Delta Q_{\text {c_mul_d }<0.5 \_3}$ is described as

$$
\begin{aligned}
\Delta Q_{\text {C_mul_d }<0.5 \_3}= & \frac{1}{8} \cdot\left\{2 \cdot(1-2 \cdot d)+\frac{d}{1-d} \cdot \frac{I_{\text {Lpp_mul }}}{I_{\text {L_mul }}}\right\}^{2} \\
& \cdot(1-d) \cdot \frac{I_{\text {L_mul }}^{2}}{I_{\text {Lpp_mul }}} \cdot T_{\mathrm{s}} \cdots \cdots \cdots(24)
\end{aligned}
$$

Then, the case of $d \geq 0.5$ is examined. Each phase of the diode current doesn't overlap and flows into the output capacitor under the condition of $d \geq 0.5$. Thus, the generated output capacitor current becomes such as Fig. 9. In this case, the waveform pattern is equal to the one of the single-phase method. However, the current which flows into the output capacitor shows the double frequency of the switching frequency and then the cycle of the waveforms becomes half because the switching pattern cycle for the switches is shifted with a $180^{\circ}$. In this moment, each peak value of the output capacitor current is derived as follows.

$$
I_{\mathrm{c} \_ \text {E_mul }}=I_{\mathrm{dp}}-I_{\mathrm{O}}
$$


Table 2. Circuit parameters (non-coupled multi-phase)

\begin{tabular}{lc|c}
\hline \hline Input voltage & $V_{\mathrm{i}}$ & $50 \mathrm{~V}$ \\
\hline Duty ratio & $d$ & $0.1 \sim 0.7$ \\
\hline Output capacitance & $C_{0}$ & $3.9 \mu \mathrm{F}$ \\
\hline Self inductance & $L_{1}, L_{2}$ & $300 \mu \mathrm{H}$ \\
\hline Output resistance & $R_{\mathrm{o}}$ & $48 \Omega$ \\
\hline
\end{tabular}



Fig. 11. Output voltage ripple of the non-coupled multiphase boost chopper circuit

$$
I_{\mathrm{c} \_ \text {F_mul }}=I_{\mathrm{dp}}-I_{\mathrm{Lpp}}-I_{\mathrm{o}}
$$

Where, $I_{\mathrm{dp}}$ is the peak value of the diode current, $I_{\mathrm{Lpp}}$ is the ripple value of the inductor current. The charge quantity of the discharge and charge time is described with these equations.

The output current $I_{\mathrm{O}}$ becomes such as the equation (18) in case of $d \geq 0.5$ as well. By means of this, from the same point of view concerning single-phase, the waveform pattern is separated as shown Fig. 10. At this point, from $I_{\mathrm{c} \_ \text {F_mul }}<0$, a range of the duty ratio, which is $d_{\text {mul_d } \geq 0.5 \_1}$, is given by

$$
d_{\text {mul_d } \geq 0.5 \_1}<\frac{1}{2}+\frac{1}{4} \cdot \frac{I_{\text {Lpp_mul }}}{I_{\text {L_mul }}}
$$

And also, from $I_{\text {c_F_mul }_{-}} \geq 0$, in case of Form2, a range of the duty ratio, which is $d_{\text {mul_d } \geq 0.5 \_2}$, is expressed as

$$
d_{\text {mul_d } \geq 0.5 \_2} \geq \frac{1}{2}+\frac{1}{4} \cdot \frac{I_{\text {Lpp_mul }}}{I_{\text {L_mul }}} .
$$

In a range of the equation (27), the range of the charge fluctuation $\Delta Q_{\text {c_mul_d } \geq 0.5 \_1}$ is represented as

$$
\begin{aligned}
\Delta Q_{\mathrm{C} \_ \text {mul_d } \geq 0.5 \_1}= & \frac{1}{8} \cdot\left(2 \cdot(2 \cdot d-1)+\frac{I_{\text {Lpp_mul }}}{I_{\text {L_mul }}}\right)^{2} \\
& \cdot(1-d) \cdot \frac{I_{\text {L_mul }}^{2}}{I_{\text {Lpp_mul }}} \cdot T_{\mathrm{s}} \cdots \cdots
\end{aligned}
$$

Likewise, in the range of equation (28), the range of the charge fluctuation $\Delta Q_{\text {c_mul_d } \geq 0.5 \_2}$ is described as

$$
\begin{aligned}
\Delta Q_{\mathrm{C} \_ \text {mul_d } \geq 0.5 \_2}= & \frac{I_{\text {Lpp_mul }}}{I_{\text {L_mul }}} \cdot(2 \cdot d-1) \cdot(1-d) \\
& \cdot \frac{I_{\text {L_mul }}^{2}}{I_{\text {Lpp_mul }}} \cdot T_{\mathrm{s}} \ldots \cdots \cdots \cdots \cdots
\end{aligned}
$$

Now, the output ripple voltage is measured and then the validity of the derived equation for the charge fluctuation is discussed. The ripple voltage $\Delta V_{\mathrm{c}}$ is expressed as the equation (11) and the theoretical value of the ripple voltage is compared with the experiment value. In this experiment, using



Fig. 12. Transformer-linked multi-phase boost chopper circuit

the current balance control, each phase of the average current is balanced ${ }^{(13)(14)}$.

The theoretical value of the output ripple voltage from the duty ratio 0.1 to 0.7 with the circuit parameters in Table 2 is depicted in Fig. 11. On the other hand, the experimental result of the output voltage ripple with the same circuit parameters is plotted in Fig. 11. From this result, in case of multi-phase method, the output ripple voltage obtains lower up to the duty ratio 0.5 gradually, and from the duty ratio 0.5 , the output ripple voltage becomes larger. Moreover, the theoretical value agrees with the experiment value closely. As a result, the validity of calculation equation concerning the quantity $\Delta Q_{\mathrm{c}}$ of the charge fluctuation for a conventional multi-phase boost chopper circuit is verified.

\section{Characteristic Analysis of Transformer- Linked Method Multi-phase Boost Chopper Circuit}

A charge characteristic analysis of the output smoothing capacitor for transformer-linked multi-phase boost chopper circuit in Fig. 12 is now discussed. In case of the transformerlinked multi-phase boost chopper circuit, the characteristic of the output capacitor current $i_{\mathrm{c}}$ changes on the border of the duty ratio 0.5 such as a conventional multi-phase method. Accordingly, separating the conditions of $d<0.5$ and $d \geq 0.5$ is required for analysis. Furthermore, each phase of the variation related to parasitic parameters doesn't exist when analyzing and also, each phase of the average current is assumed to be equal.

Firstly, the case of $d<0.5$ is investigated. Assuming that each phase of the diode current are $i_{\mathrm{d} 1}$ and $i_{\mathrm{d} 2}$ respectively, the output current waveform with $d<0.5$ is described as Fig. 13. In case of transformer-linked method, the inductor current is changed by the influence of each phase of the switching operation because the inductors of each phase are linked by the transformer. In this paper, the width of the inductor current is assumed as complete twice frequency current for making the analysis simple. It is understood that each of the diode current overlaps and the output capacitor current is generated under the condition of $d<0.5$ in Fig. 13. In this time, each peak value of the output capacitor current is as follows.

$$
\begin{aligned}
& I_{\mathrm{c}_{-} \mathrm{A} \_\mathrm{mtl}}=2 I_{\mathrm{dp}}-I_{\mathrm{o}} \ldots \ldots \ldots \ldots \ldots \ldots \ldots \ldots \ldots \ldots \ldots \\
& I_{\mathrm{c} \_\mathrm{B} \_\mathrm{mtl}}=2 I_{\mathrm{dp}}-2 I_{\mathrm{Lpp}}-I_{\mathrm{O}} \\
& I_{\mathrm{c} \_\mathrm{C} \_\mathrm{mtl}}=I_{\mathrm{dp}}-I_{\mathrm{Lpp}}-I_{\mathrm{O}} \\
& I_{\text {c_D_mtl }}=I_{\text {dp }}-I_{\mathrm{O}}
\end{aligned}
$$

Where, $I_{\mathrm{dp}}$ is the peak value of the diode current, $I_{\mathrm{Lpp}}$ is the ripple value of the inductor current. The charge quantity of the discharge and charge time can be derived with these 


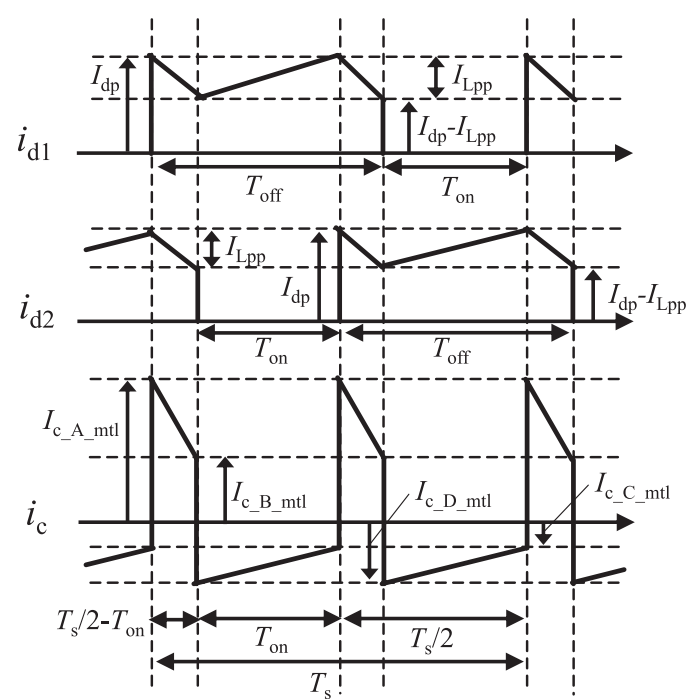

Fig. 13. Current waveforms of transformer-linked multiphase boost chopper circuit $(d<0.5)$



Form 1

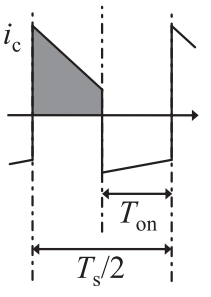

Form 2

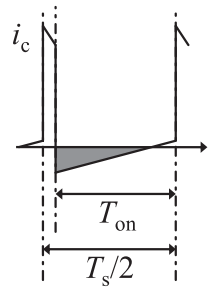

Form 3
Fig. 14. Output capacitor current waveforms of the transformer-linked multi-phase boost chopper circuit $(d<0.5)$

equations. At this point, the time integrated values of the current $i_{\mathrm{d}}$ which is supplied from the diode and current which is outputted to the load for one switching cycle became equal. That's why the equation is the same as the equation (16). Furthermore, using the relational equation (17), the output capacitor current $I_{\mathrm{o}}$ of transformer-linked method is the same as the equation (18) as with a conventional method. From the same point of view such as single-phase method, the waveform pattern is divided to three forms as shown in Fig. 14. Form 1 is described that the case of a calculation part of the positive current is a triangle shape, Form 2 is defined that the case of a calculation part of the positive current is a trapezoid shape, and Form 3 is that the case of a calculation part of the negative current is a triangle shape respectively. In case of Form1, a range of the duty ratio, $d_{\mathrm{mtl} \mathrm{d}_{\mathrm{d}}<0.5_{-} 1}$ is as follows from $I_{\text {c_B_Bntl }}<0$.

$$
d_{\mathrm{mtl} \_\mathrm{d}<0.5 \_1}<\frac{1}{2} \cdot \frac{I_{\text {Lpp_mtl }}}{I_{\text {L_mtl }}} .
$$

The condition which the waveform changes is determined by the ratio of the average inductor current $I_{\mathrm{L} \_\mathrm{mtl}}$ to the ripple current's amplitude $I_{\text {Lpp_mtl }}, I_{\text {Lpp_mtl }} / I_{\text {L_mtl }}$. In case of Form 3 , a range of the duty ratio, $d_{\mathrm{mtl} \_\mathrm{d}<0.55_{3} 3}$ is as follows from $I_{\text {c_C_mtl }} \geq 0$

$$
d_{\mathrm{mtl} \_\mathrm{d}<0.5 \_3} \geq \frac{1}{2}-\frac{1}{4} \cdot \frac{I_{\text {Lpp_mtl }}}{I_{\mathrm{L} \_\mathrm{mtl}}} .
$$

A range of Form 2 exists in the field between Form 2 and
Table 3. Circuit parameters (transformer-linked multiphase)



Fig. 15. Output voltage ripple of the transformer-linked multi-phase boost chopper circuit

Form3. Accordingly, a range of the duty ratio, $d_{\mathrm{mtl} \_\mathrm{d}<0.5 \_2}$ is given by

$$
\frac{1}{2} \cdot \frac{I_{\mathrm{Lpp} \_\mathrm{mtl}}}{I_{\mathrm{L} \_\mathrm{mtl}}} \leq d_{\mathrm{mtl} \_\mathrm{d}<0.5 \_2}<\frac{1}{2}-\frac{1}{4} \cdot \frac{I_{\mathrm{Lpp} \_\mathrm{mtl}}}{I_{\mathrm{L} \_\mathrm{mtl}}} \cdots \cdots
$$

Next, the quantity of the charge fluctuation $\Delta Q_{\mathrm{c}}$ is derived with these three ranges. In the range of equation (35) where the waveform of Form 1 is formed, the quantity of the charge fluctuation $\Delta Q_{\text {c_mtl_d }<0.5 \_1}$ is expressed as

$$
\begin{aligned}
\Delta Q_{\text {C_mtl_d }<0.5 \_1}= & \frac{1}{4} \cdot\left(2 \cdot d+\frac{I_{\text {Lpp_mtl }}}{I_{\text {L_mtl }}}\right)^{2} \\
& \cdot\left(\frac{1}{2}-d\right) \cdot \frac{I_{\mathrm{L} \_\mathrm{mtl}}^{2}}{I_{\mathrm{Lpp} \_\mathrm{mtl}}} \cdot T_{\mathrm{s}}
\end{aligned}
$$

Likewise, in the range of equation (37) where waveform of Form 2 is formed, the quantity of the charge fluctuation $\Delta Q_{\text {c_mtl_d }<0.5 \_2}$ is represented as

$$
\Delta Q_{\mathrm{C} \_\mathrm{mtl} \_\mathrm{d}<0.5 \_2}=\frac{I_{\mathrm{Lpp} \_\mathrm{mtl}}}{I_{\mathrm{L} \_\mathrm{mtl}}} \cdot(1-2 \cdot d) \cdot d \cdot \frac{I_{\mathrm{L} \_\mathrm{mtl}}^{2}}{I_{\mathrm{Lpp} \_\mathrm{mtl}}} \cdot T_{\mathrm{s}}
$$

And also, in the range of equation (36) where waveform of Form 3 is formed, the quantity of the charge fluctuation $\Delta Q_{\mathrm{c} \_\mathrm{mtl} \_\mathrm{d}<0.5 \_3}$ is described as

$$
\begin{aligned}
\Delta Q_{\mathrm{C} \_ \text {mtl_d }<0.5 \_3}= & \frac{1}{8} \cdot\left\{2 \cdot(1-2 \cdot d)+\frac{I_{\mathrm{Lpp} \_\mathrm{mtl}}}{I_{\mathrm{L} \_\mathrm{mtl}}}\right\}^{2} \\
& \cdot d \cdot \frac{I_{\mathrm{L} \_\mathrm{mtl}}^{2}}{I_{\mathrm{Lpp} \_\mathrm{mtl}}} \cdot T_{\mathrm{s}} \cdots \cdots \cdots \cdots \cdots
\end{aligned}
$$

Each phase of the diode current doesn't overlap and flows into the output capacitor under the condition of $d \geq 0.5$. Thus, the current waveforms of transformer-linked method are also the same as a conventional multi-phase boost chopper. The quantity of the charge fluctuation and the field division related to the charge calculation is the same as a conventional method as well. For that reason, it is understood 
Table 4. Relational equations of charge fluctuation (In case of $I_{\mathrm{Lpp}} / I_{\mathrm{L}}=1 / 2$ )

\begin{tabular}{|c|c|c|}
\hline Circuit topology & Duty range & $\begin{array}{l}\text { Equation of the } \\
\text { charge fluctuation }\end{array}$ \\
\hline \multirow{2}{*}{$\begin{array}{c}\text { Single-phase } \\
\text { boost chopper circuit }\end{array}$} & $d<0.25$ (Form 1 ) & (9) \\
\hline & $d \geq 0.25$ (Form 2 ) & $(10)$ \\
\hline \multirow{5}{*}{$\begin{array}{c}\text { Multi-phase } \\
\text { boost chopper circuit } \\
\text { (Non-coupled method) }\end{array}$} & $d<0.11$ (Form1) & (22) \\
\hline & $0.11 \leq d<0.41$ (Form 2 ) & (23) \\
\hline & $0.41 \leq d<0.5$ (Form 3 ) & (24) \\
\hline & $0.5 \leq d<0.63$ (Form 1 ) & (29) \\
\hline & $0.63 \leq d$ (Form 2$)$ & (30) \\
\hline \multirow{5}{*}{$\begin{array}{c}\text { Multi-phase } \\
\text { boost chopper circuit } \\
\text { (Transformer-linked } \\
\text { method) }\end{array}$} & $d<0.25$ (Form 1 ) & $(38)$ \\
\hline & $0.25 \leq d<0.38$ (Form 2 ) & (39) \\
\hline & $0.38 \leq d<0.5$ (Form 3 ) & $(40)$ \\
\hline & $0.5 \leq d<0.63$ (Form 1 ) & (29) \\
\hline & $0.63 \leq d$ (Form 2$)$ & $(30)$ \\
\hline
\end{tabular}

that the quantity $\Delta Q_{\mathrm{c} \_\mathrm{mtl} \_\mathrm{d} \geq 0.5 \_1}$ of the charge fluctuation on transformer-linked method shows the same calculation results as the equation (29). In the same way, the equation for the quantity $\Delta Q_{\text {c_mtl_d } \geq 0.5 \_2}$ of the charge fluctuation is equal to a conventional equation (30).

The output ripple voltage is measured from the experiment and the validity of the derived equation is now discussed. The ripple voltage $\Delta V_{\mathrm{c}}$ is expressed as the equation (11), and then the theoretical value is compared with the measurement value. In this experiment, using the current balance control $^{(13)(14)}$, each phase of the average current is balanced. The theoretical value of the output ripple voltage from the duty ratio 0.1 to 0.7 with the circuit parameters in Table 3 is depicted in Fig. 15. On the other hand, the experimental result of the output voltage ripple with the same circuit parameters is plotted in Fig. 15. From this result, in case of multi-phase method, the output ripple voltage becomes lower up to the duty ratio 0.5 gradually, and then from the duty ratio 0.5 , the output ripple voltage becomes larger.

Moreover, the theoretical value agrees with the experiment value closely. As a result, the validity of calculation equation concerning the quantity $\Delta Q_{\mathrm{c}}$ of the charge fluctuation for the transformer-linked multi-phase boost chopper circuit is verified.

\section{Comparison of Voltage Ripple Characteristic}

Characteristic evaluation concerning downsizing of the output capacitor is now discussed by comparing the voltage ripple characteristic of three circuit topology which the validity is verified from the experiment. In this section, the relative evaluation between multi-phase method's output capacitor for downsizing is carried out. And meanwhile, the difference between conventional multi-phase method and transformerlinked multi-phase method is investigated. The comparison condition is that the ratio $I_{\mathrm{Lpp}} / I_{\mathrm{L}}$ of inductor current ripple is $1 / 2$, switching frequency $f_{s}$ is $50 \mathrm{kHz}\left(T_{\mathrm{s}}=20 \mu \mathrm{s}\right)$, output current $I_{\mathrm{o}}$ is $10 \mathrm{~A}$, and output capacitance $C_{\mathrm{o}}=10 \mu \mathrm{F}$ for three circuit topology. We have to go through three steps in order to compare the capacitor voltage ripple of each circuit topology. Firstly, it is necessary to derive the range of the



Fig. 16. Comparison result for voltage ripple characteristic of the output capacitor (absolute evaluation)



Fig. 17. Comparison result for voltage ripple characteristic of the output capacitor (relative comparison)

duty ratio corresponding to the charge fluctuation of the capacitor. For instance, the boundary of Form 1 and Form 2 is $d=0.25$ when we substitute $I_{\mathrm{Lpp}} / I_{\mathrm{L}}=1 / 2$ for equations (7), (8) of the single phase method.

Secondly, the equations (9), (10) and (6) are applied on the boundary of this duty ratio respectively in case of singlephase method, and the quantity $\Delta Q_{\mathrm{c}}$ of the charge fluctuation is calculated. (The equations of the charge fluctuation corresponding to each duty ratio range in each method are shown in Table 4).

Finally, the voltage ripple $\Delta V_{\mathrm{c}}$ is expressed from equation (11). Likewise, when this process is applied to the noncoupled and transformer-linked methods respectively, we can compare the voltage ripple $\Delta V_{\mathrm{c}}$. Based on the above the procedure, the result of absolute comparison for the voltage ripple characteristics of output capacitor among three circuit topology are shown in Fig. 16. From this figure, the amplitude of the output voltage ripples concerning the non-coupled and the transformer-linked methods are suppressed as compared with single-phase method in the all duty range. And also, to compare three methods relatively, the characteristic of ratio of the output voltage amplitude $\Delta V_{\mathrm{o}_{-} \mathrm{mul}} / \Delta V_{\mathrm{o}_{-} \sin }$ is shown in Fig. 17. This is the characteristic single-phase and conventional multi-phase methods to the duty ratio. Likewise, the characteristic of the amplitude of the output voltage ripple regarding single-phase and transformer-linked multiphase methods to the duty ratio is plotted in Fig. 17. As seen in Fig. 17, the amplitude of the output voltage ripple con- 
cerning the non-coupled method and the transformer-linked method are suppressed less than half of single-phase method in the all duty range. Furthermore, it is confirmed that the reduction of the amplitude of the output voltage ripple less than one-tenth around the duty ratio 0.5 . And also, the characteristic which conventional method of the amplitude of the output voltage ripple becomes smaller than transformer-linked method is described in the portion of the range less than the duty ratio 0.5 . This reason is the equations (22) and (38), (24) and (40) are different on the result of analysis concerning the charge fluctuation on the output capacitor. In the duty ratio less than 0.5 , the quantity of the charge fluctuation on the output capacitor depends on the ripple form of the flowing diode current because the each phase of the diode current overlaps. The diode current of transformer-linked method in Fig. 13 is affected by applying the coupled inductor and each phase of the switching operation. Owing to this reason, on the non-coupled and the transformer-linked methods, the ripple forms of the diode currents which flow through the output capacitor are different even though their ripple ratio is corresponded. Especially, this difference of the ripple form influences to the peak value of the output capacitor's current. The current $I_{\text {c_A_mul }}$ of the non-coupled method obtains larger under the condition which the ripple ratio is the same even when comparing the current $I_{\text {c_A_mtl }}$ of the transformerlinked method. Accordingly, the quantity of the charge fluctuation of the Form 1 of non-coupled and transformer-linked methods derive from the current $I_{\mathrm{c} \_ \text {A_mul }}$ and $I_{\mathrm{c} \_ \text {A_m }}$ ml so that the disparity to the quantity of the charge fluctuation occurs such as the equations (22) and (38) in the same multi-phase method. Likewise, the disparity of the current $I_{\text {c_C_mul }}$ of non-coupled method and $I_{\text {c_C_mtl }}$ of the transformer-linked method due to the difference ripple form of the diode current between the non-coupled and transformer-linked methods. Consequently, it is considered that there is the different quantity of the charge fluctuation between Form 3 of the non-coupled and transformer-linked methods. These different characteristic is not much bigger and a factor for denying usefulness of transformer-linked method.

\section{Conclusions}

The miniaturization possibility of the output capacitor in the transformer-linked method boost chopper system is evaluated and discussed in this paper. From the comparative voltage ripple analysis of the output capacitor for the conventional non-coupled method and the transformer-linked method, it can be conclude that there is no difference of the output capacitor size in each type of converter from same capacitance value point of view. The experimental ratio data of the capacitor charge ripple suggest that the transformerlinked method power converter can achieve higher power density because of the performance of the downsized capacitor as compared with the conventional non-coupled method.

\section{References}

( 1 ) M. Hirakawa, M. Nagano, Y. Watanabe, K. Andoh, S. Nakatomi, and S. Hashino: "High Power Density DC/DC Converter using the CloseCoupled Inductors", the 1st IEEE Energy Conversion Congress and Exposition (ECCE2009), pp.1760-1767 (2009)
( 2 ) L. Yan and B. Lehman: "An Integrated Magnetic Isolated Two-Inductor Boost Converter: Analysis, Design and Experimentation", IEEE Trans. Power Electronics, Vol.20, No.2, pp.332-342 (2005)

( 3 ) L. Solero, A. Lidozzi, and J.A. Pomilio: "Design of Multiple-Input Power Converter for Hybrid Vehicles", IEEE Trans. Power Electronics, Vol.20, No.5, pp.1007-1016 (2005)

( 4 ) E. Laboure, A. Cuniere, T.A. Meynard, F. Forest, and E. Sarraute: "A Theoretical Approach to InterCell Transformers, Application to Interleaved Converters", IEEE Trans. Power Electronics, Vol.23, No.1, pp.464-474 (2008)

( 5 ) A. Fratta, P. Guglielmi, G.M. Pellegrino, F. Scapino, and F. Villata: "Efficient Modulation Technique and NL Control of H-bridge Boost Converters for Battery-Supplied AC Motor Drives", IEEE International Symposium on Industrial Electronics, Vol.2, pp.806-812 (1999)

( 6 ) J. Imaoka, Y. Ishikura, T. Kawashima, and M. Yamamoto, "Optimal Design Method for Interleaved Single-phase PFC Converter with Coupled Inductor", Record of IEEE Energy Conversion Congress \& Expo (ECCE), pp.18071812 (2011)

( 7 ) M. Nakahama, M. Yamamoto, and Y. Satake: "Trans-linked Multi-phase Boost Converter for Electric Vehicle", Record of IEEE Energy Conversion Congress \& Expo (ECCE), pp.2458-2463 (2010)

( 8 ) J.C. Schroeder and F.W. Fuchs: "Detailed Characterization of Coupled Inductors in Interleaved Converters Regarding the Demand for Additional Filtering" IEEE Energy Conversion Congress and Expo (ECCE2012), pp.759-766 (2012)

( 9 ) J. Imaoka and M.Yamamoto: "A Novel integrated magnetic core structure suitable for Transformer-Linked Interleaved Boost Chopper Circuit" IEEE Energy Conversion Congress and Expo (ECCE2012), pp.3279-3284 (2012)

(10) K. Hartnett, J. Hayes, and M. Egan: "Novel CCTT-Core Split-Winding Integrated Magnetic for High-power DC-DC Converters", IEEE Energy Conversion Congress and Expo (ECCE2011), pp.598-605 (2011)

(11) J. Zhu and A. Pratt: "Capacitor Ripple Current in an Interleaved PFC Converter", IEEE Trans. Power Electronics, Vol.24, pp.1506-1514 (2008)

(12) J. Imaoka, T. Kawashima, M. Yamamoto, H. Asuke, H. Terui, and S. Takano: "Examination about Design of Multi-Phase Boost Chopper Circuit Using Mutual Coupled Inductors", The 2010 Japan Industry Applications Society Conference, Vol.1, pp.201-204 (2010) (in Japanese)

(13) M.L. Bolloch, M. Cousineau, and T. Meynard: "Current-sharing control technique for interleaving VRMs using intercell transformers", IEEE European Conference on Power Electronics and Applications (EPE2009), pp.1-10 (2009)

(14) Y. Nakamura and M. Yamamoto: "Optimal control design method for translinked type multi-phase boost converter", IEEE International Conference on Power Electronics and Drive Systems, pp.901-904 (2011)

Jun Imaoka (Student Member) received the B.S. and M.S. degrees


in electrical and electronic engineering from the Shimane University, Shimane, Japan, in 2011 and 2013 respectively. $\mathrm{He}$ is currently working toward $\mathrm{Ph} . \mathrm{D}$. degree in electrical and electronic engineering in Shimane University, Shimane, Japan. His research interests include design of magnetic components and modeling for high power density DC/DC converters and $\mathrm{AC} / \mathrm{DC}$ converters.

Masayoshi Yamamoto (Member) received the M.S. and Ph.D. degree

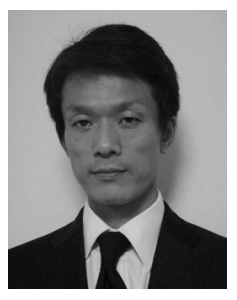
in science and engineering from Yamaguchi University, Yamaguchi, Japan in 2000 and 2004 respectively. From 2004 to 2005, he was with Sanken Electric Co., Ltd., Saitama, Japan. Since 2006, he has been with Shimane University, Shimane, Japan. His research interests include power supply for HEV (boost converter, buck converter, 3-phase inverter, and digital control), charging system for EV, LED illumination system for a tunnel, EMI of switching power supply, wireless power transfer etc. 
Yuta Nakamura (Member) received the B.S. and M.S. degrees in

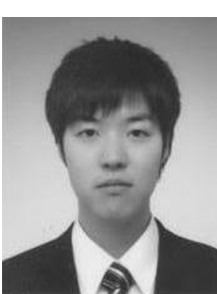
electrical and electronic engineering from the Shimane University, Shimane, Japan, in 2010 and 2012 respectively. Since 2012, He has been with DENSO CORPORATION, Aichi, Japan. His research interests include modeling for DC/DC converter, design of digital and analog control for power converters.
Takahiro Kawashima (Member) received the M.S. and Ph.D. de-
grees in electrical and electronic engineering from grees in electrical and electronic engineering from
the Shimane University, Shimane, Japan, in 2009 and
2012 respectively. He is currently a technical researcher at the Shimane Institute for Industrial Technology. His research interests include high power density power converter, design of magnetic components, EMC problems in power electronics and softswitching techniques. 